\title{
An Automatic Model for Brain Tumor Detection using Machine Learning Techniques
}

\author{
C.Gunasundari, R.Punidha
}

\begin{abstract}
- machine acing is a most recent technique that is connected in different essential projects; directly here we're connected to find the example dependent on the given preparing records. on this paper, we proposed a framework examining procedure to blast a strategy for identifying tumor zone from the given personality MRI depictions. The psyche is a central organ in our body; it controls the whole edge arrangement of a human. there can be a wild and mind boggling increment of cells in tissues makes most tumors in the body. Our contemplations is encased by an unyielding skull, there can be any undesirable blast in that amounts makes an issue. Benevolent and harmful are two sorts of tumor in our psyche, The Benign tumor is non-destructive and the dangerous tumor is carcinogenic likewise hazardous infection. Glioma is one of the mind tumors that is begun from the glial cells of the considerations or spine. principally dependent on the overview 80 level of the tumor is dangerous and unquestionably speedy future. Attractive Resonance Imaging is a way to supply the MRI previews of the cerebrum, this brings to the table additional actualities about the psyche. fundamentally based at the MRI picture records the treatment for tumor sufferers has been arranged. mind tumor photograph division and location of the tumor territory are not the perfect techniques by method for guide. on this paper, we've proposed a programmed and effective methodology for distinguishing tumor from the MRI previews utilizing a gadget examining procedure. a robotized strategy to segregate the mind tumor region from given MRI dependent on guide Vector device(SVM) and it is done $96.2 \%$ accuracy,94.1percentspecificity, and 97\%sensitivity, the outcomes shows that the higher presentation..
\end{abstract}

Keywords: Amino acids, antigenicity, normalization and Protein modeling Classification,Brain Tumor, SVM, FBB, GLCM, MRI.

\section{INTRODUCTION}

These days, device mastering model has been developed for precise applications like pix, agribusiness, concoction depictions, materials, casual businesses, and cash related applications and so forth. Restorative photo investigation assumes noteworthy undertaking inside the ongoing scenario. Our reason is to accumulate an effective programmed version for thoughts tumor discovery from the given MRI images.

This investigation portrays the troubles of department of ordinary and weird tissues from the MRI snap shots using

spotlight extraction strategies and classifier of SVM[1,2]. Cerebrum tumor is one reason for the excessive brevity of the overall populace. the ordinary drugs for the mind tumor are chemotherapy and radiological treatment or the blends of each. In mild of unmatched sensitive tissue separation, high spatial goals, differentiate and not the use of destructive ionizing radiation, MRI approach is the preceding device within the clinical and cautious situation. The thoughts is imaged based totally at the thickness of water in delicate tissue this is better contrasted with tissues like bone in the MRI technique[3].

rather than medical manner, the lifestyles structures of the mind may be visible through the radiologists via the MRI photos. via MRI images no character however we cannot diagnosethe cerebrum bodily and it may lead the misdiagnosing. Likewise, the various complex attributes of cerebrum tumor make willpower further complicated [4]. Thusly, a pc Aided Diagnostic (CAD) framework is straightforward as a way to help radiologist in spotting and characterizing cerebrum tumors. For this ShanShen et al proposed fluffy c manner grouping (IFCM). The proposed calculation is predicated upon on location fascination. this is considered many of the close by pixels. This place fascination relies upon the pixel forces, the spatial characteristic of the neighbor pixels and on neighborhood pixel structure. The grouping of the thoughts tumor is made with the help of the faux neural device (ANN) in light of the examination among spotlight vectors [5].In ebb and drift years, analysts have proposed a ton of things of view for this goal, which live into classifications. One is administered affiliation, consisting of bolster vector device (SVM) [6] and ok closest pals (good enough-NN) [7]. the opportunity one is solo characterization [8], together with self-affiliation spotlight map (SOFM) [6] and fluffy c-implies [9]. no matter the truth that each one of these strategies produce exquisite outcomes, the directed classifier plays regular than unaided classifier in mild of arrangement precision (success in characterization price). Be that as it could, the characterization correctnesses of most current strategies have been no longer higher than ninety five \%.finally the purpose of this paper is to discover the extra real machine. In managed order, the SVMs are brilliant in magnificence characterization techniques depending on the AI speculation [10-12]. SVMs have noteworthy favorable instances of India.(email: gunasundari.cs@gmail.com)

Dr.R.Punidha, Professor, Bharathiyar Instittute of Engineering for Women Tamilnadu, India.(email: punidhar@gmail.com) 
excessive exactness, great numerical tractability, and direct geometric understanding contrasted with certainly one of a type strategies like counterfeit neural gadget, preference tree, and Bayesian tool. similarly, it want no longer hassle with an large huge kind of making prepared tests to hold a strategic distance from over turning into [13]. every other technique is supplied right here via becoming a member of the SVM and ANN for tumor association. Cerebrum MRI snap shots with everyday and irregular physical games are earlier than the whole thing upgraded thru some channel and preprocessing steps. along the ones traces, for the identity and arrangement of the cerebrum tumor the joined division paperwork to be unique temper primarily based $\mathrm{k}$ implies and changed Fuzzy C-implies (TKFCM) grouping calculation [14] is attached.

on this paper, a programmed technique is proposed the usage of AI machine and applied as a classifier to perceive and seclude tumor from ordinary thoughts MRI pics with SVM. Portioned snap shots are superior utilising improve structures, for example, differentiate improvement and mid-bypass expand. The test outcomes are carried out ninety six. $2 \%$ exactness, ninety four. $1 \%$ particularity, and 90 seven $\%$ affectability. it is showing the viability of the proposed technique for distinguishing commonplace and abnormal tissues from cerebrum MR photos.

\section{II.METHODOLOGY}

This part consists of the overall methodology and steps followed for detecting from the given Brain MRI.

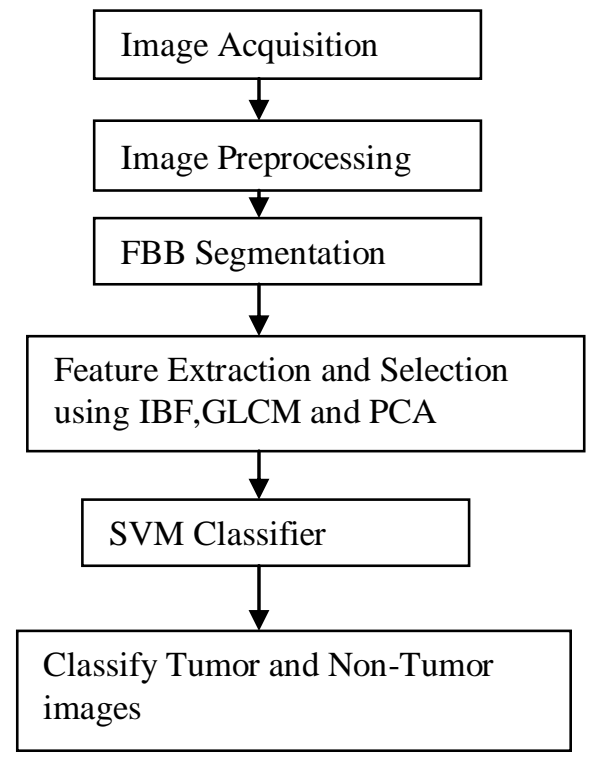

Fig.1 Proposed model block diagram

\section{A. Image Data Collection}

The initial step for figuring out tumor from the thoughts MRI is the gathering pictures. The snap shots had been taken from the internet open property. The vault of MRI photos consists of ordinary and tumor induced pix like terrible outstanding glioma and high-grade gliomas[13]-[15]. For the grouping of tumor detection50 MRI images had been took.

\section{B. Photograph Pre-Dealing With}

the following degree for characterizing tumor is preprocessing the snap shots which might be recovered from the open save of net assets. Preprocessing step, it's beneficial for upgrading the parameters beautify the visible look of MR snap shots. The technique signal to commotion proportion improves the lucidity of the crude photograph.getting rid of the unessential clamor out of sight of the image and undesired elements. Smoothening is the method used to clean the locale's inner issue and guard image edges. The proposed approach for this paper is anisotropic diffusion[24] is a way for diminishing commotion without evacuating vital substance like edges, traces, and so forth to be acquainted with investigating the picture. Anisotropic dispersion appearance loves the manner that makes a scale space, in which a image produces a parameterized group of progressively an ever developing amount of obscured pix dependent on a dissemination approach. each one of the subsequent images on this circle of relatives is given as a convolution among the photo and a second isotropic Gaussian channel, in which the width of the channel increments with the parameter. This dissemination procedure is a immediately and area-invariant exchange of the first image.

\section{Cranium Stripping}

cranium stripping[25]is a approach used to expel non-cerebrum tissues, for example, the scalp, fats, eyes, neck, and so on from thegiven input MR images. This strategy is useful to improve the speed and exactness of recuperation picture identity and prediction. The proposed method is twofold thresholding department method to change over our information picture into a parallel form of the photo which does not contain the unwanted tissues subtleties are not on hand. in this twofold thresholding method, better and reduce edges have been considered due to the fact the preliminary step is disintegration for evacuating the cranium divide for that we are accepting 3 because the span for expelling undesirable components. next, district filling calculation for filling the openings in the MR pictures. At lengthy final, the associated foundation pixels are modified to frontal area pixels with the goal that the gaps in cerebrum MR images were evacuated.

The disintegration of a paired photo $f$ through an organizing aspect $s$ (alluded to af $s$ ) produces a few different parallel picture. $\mathrm{g}=\mathrm{f} \mathrm{s}$ with those the least bit regions $(\mathrm{x}, \mathrm{y})$ in which the organizing element s suits the records photo $f$, as an instance $\mathrm{g}(\mathrm{x}, \mathrm{y})=1$ suits $\mathrm{f}$ and 0 normally, rehashing for all pixel arranges $(x, y)$.

The increase of a picture $f$ with the aid of an organizing element $\mathrm{s}$ (alluded to as fs) makes another twofold imageg $=\mathrm{f}$ $\mathrm{s}$ with those at all areas $(\mathrm{x}, \mathrm{y})$ of the inception of an organizing aspect in which that organizing aspect $s$ hits the records picture $f$, for example $g(x, y)=1$ if $s$ hits $f$ and 0 typically, rehashing for all pixel organizes $(\mathrm{x}, \mathrm{y})$. expansion has the opposite impact to disintegration - it consists of a layer of pixels to every the internal and out of doors limits of areas.

\section{Characteristic Extraction}

GLCM framework mirrors the association in the area among the pixels. thru constructing grey diploma

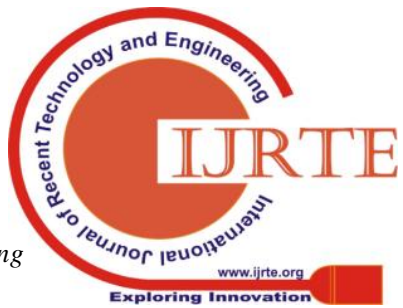


Co-occasion Matrix, it indicates the connections among pixels in the district. The GLCM depends on a surmising of the second-request common confined danger $\mathrm{d}, \theta$ )for a variety $=0,45$, ninety, $135^{\circ}$, and so on., and never like separations, $d=1,2$, three, four, and 5. The $p(i, j, d)$ encompass is the probability of getting a darkish degree I and j for 2 pixels, which can be placed with an intersample increase $d$ and a course $\theta$.

As some distance as variety $\mathrm{d}$ and mind-set $\theta$, the spatial association is depicted. The pair of pixels at separation $d$ ought to have a similar dark qualities if the floor is coarse and separation $d$ is little. but, the couple of pixels at separation $d$ want to often be very brilliant for a high-quality surface, so the motivation in the GLCM need to be extended moderateand even[22].

force primarily based absolutely trends (IBF) acquired 5 attributes to be precise mean, state-of-the-art deviation, coarseness, skewness, and kurtosis. The underlying and divided image separates the mixture of 10 functions. The classifier likewise uses the location of the extricated tumor and shakers coefficient similitude record to institution the forms of tumors. thru the evaluation, it is found that the classifier's presentation parameters, as an example, precision, explicitness, and affectability are advanced with the aid of considering the tumor locale and bones coefficient as a superb element of the highlights.

The features are calculated based on these formulas

$$
\begin{aligned}
& \text { Energy }=\sum_{i, j=0}^{N-1}\left(P_{i j}\right)^{2} \\
& \text { Entropy }=\sum_{i, j=0}^{N-1}-\ln \left(P_{i j}\right) P_{i j} \\
& \text { Contrast }=\sum_{i, j=0}^{N-1} P_{i j}(i-j)^{2} \\
& \text { Correlation }=\sum_{i, j=0}^{N-1} P_{i j} \frac{(i-\mu)(j-\mu)}{\sigma^{2}} \\
& \text { Homogeneity }=\sum_{i, j=0}^{N-1} \frac{P_{i j}}{1+(i-j)^{2}} \\
& \text { Mean }\left(\mu_{i}\right)=\sum_{i, j=0}^{N-1} i P_{i j} \quad \operatorname{Mean}\left(\mu_{j}\right)=\sum_{i, j=0}^{N-1} j P_{i j} \\
& \quad \operatorname{Variance}\left(\sigma_{i}^{2}\right)=\sum_{i, j=0}^{N-1} P_{i j}(i-\mu)^{2} \\
& \text { Variance }\left(\sigma_{j}^{2}\right)=\sum_{i, j=0}^{N-1} P_{i j}(j-\mu)^{2} \\
& \text { Standard Deviation }\left(\sigma_{i}^{2}\right)=\sqrt[2]{\sigma_{i}} \\
& \quad \text { Standard Deviation }\left(\sigma_{j}^{2}\right)=\sqrt[2]{\sigma_{j}} \\
& \text { Dissimilarity= }=\sum_{i, j=0}^{N-1} P_{i j}|i-j| \\
& \text { The capacities separated from the GLCM, SFTA, and IBF }
\end{aligned}
$$
approach are genuinely to be advanced the use of highlight decision to allow the classifier to order tumor type utilizing best pertinent abilities. The greatest fundamental thing assessment (PCA) is utilized best to select out pertinent capacities that licenses you to streamline the classifier's estimation unpredictability. In the event that trademark determination isn't completed, scientific calculations on the classifier might be improved, as a way to diminish the classifier's intricacy, applicable abilities should be extricated ahead of time than the classifier strategies them. The objective of the PCA is depicted as pursues: 1. get rid of shutting propensities to characterize best appropriate attributes from the monstrous arrangement of trademark vector. 2. contingent upon the hugeness to find new abilities. three. to concentrate on contrasts in practically identical attributes and to convey best incredible styles dependent on the best change. 4.Optimize the capacities picked.

\section{E. Photograph Segmentation}

picture division is a pivotal advance for musings tumor discovery model, it isolates the arrangement of pixels into an a set principally dependent on homogeneous houses including profundity, power, tinge or surface.

The yield of the image division step is to name to see the homogeneous area or an immovable of shapes to give a clarification for the limits of the district. inside the division step yield is to isolate the photo components dependent on the tissue types which comprise of white be counted(WM),grey depend assortment

(GM), and cerebrospinal fluid(CSF).The outcomes of the division step is additionally utilized for perusing anatomical structures, find the neurotic zones, treatment of careful arranging and for imagining. there are different division methodologies are accessible, they are thresholding based absolutely, area developing, water sheds and lines based absolutely strategies. During the ones contemporary systems issues had been amended through the proposed methodology. at the preprocessing degree, to separate the insights in regards to tumor from the info MRI photo then additional bits of skull certainties had been killed, after that the utilization of anisotropic dissemination channel is connected to discard clamor. to utilize quick Bounding field set of rules(FBB)[20] into that photo and it recovers the tumor spot jumped using the bouncing box and the vital part in that case example factors for preparing of SVM classifier.

quick jumping field[20 ] works in steps.To get pivot parallel square shapes, the idea set of second MR cuts is at first managed by and by. at last, all bouncing pressing holders are gathe red to comprehend the tumor or edema that is essentially located.those stages are portrayed inside the subdivisions underneath. Presently in this zone supply the major statute ahead of time of FBB: an adjustment revelation code, wherever a district of exchange (D) is watched playing out an investigate photograph $(\mathrm{Im})$, when connected through a reference picture $(\mathrm{Rm})$.In $\mathrm{FBB}$, the symmetry arrangement is later overseen by means of a pivotal MR stage, the left (or right) halfway guides with the guide of the photo investigate, and the best possible (or left) 1/2 of conveyances with the asset of the photograph reference R..right here, the spot of progress $\mathrm{D}$ stays confined close to a hub parallel square shape that is basically proposed to find irregularities. This method keeps up never again like more often than not arranged item discovering methodologies in that we secure this change as a region based unquestionably broad transformation that is altered as most systems that see the trade as an area pixel-to-pixel deviation.At this certificate, tumor is spoken to as the locale of progress inside the check photo and all intracranial tissue increments separated from tumor are spoken to because of the reality the region of no exchange. We utilize a simple rating highlight that could surely understood the other area D with two right unmarried fast appraisals by the upstanding picture track and the extra nearby the level music. The rectangle region is defined as

$\mathrm{D}=\left[\mathrm{L}_{\mathrm{X}}, \mathrm{L}_{\mathrm{y}}\right] \times\left[\mathrm{L}_{\mathrm{y}}, \mathrm{U}_{\mathrm{y}}\right]$

$\mathrm{D}$ denotes tumor comprising region. $\mathrm{T}(\mathrm{I})$ is the top

Published By 
sub-rectangle and $\mathrm{B}(\mathrm{I})$ is the bottom sub-rectangle of the image. $\mathrm{I}$ is the midpoint of the image. Score function used to discover the values of Ly and Uy in a vertical sweep.

$$
\mathrm{E}(\mathrm{I})=\mathrm{BC}\left(\mathrm{P}_{\mathrm{I}}^{\mathrm{T}(\mathrm{I})}, \mathrm{P}_{\mathrm{R}}^{\mathrm{T}(\mathrm{I})}\right)-\mathrm{BC}\left(\mathrm{P}_{\mathrm{I}}^{\mathrm{B}(\mathrm{I})}, \mathrm{P}_{\mathrm{R}}^{\mathrm{B}(\mathrm{I})}\right)
$$

Where $\mathrm{P}_{\mathrm{I}}^{\mathrm{T}(\mathrm{I})}$ denotes normalized intensity histogram of the region $\mathrm{T}(\mathrm{I})$ in the test image. $\mathrm{P}_{\mathrm{R}}{ }^{\mathrm{T}(\mathrm{I})}, \mathrm{P}_{\mathrm{I}}{ }^{\mathrm{B}(\mathrm{I})}, \mathrm{P}_{\mathrm{R}}{ }^{\mathrm{B}(\mathrm{I})}$ are defined accordingly.E(I) is the score function.

Bhattarcharya coefficient is defined as

\section{$\mathrm{BC}(\mathrm{a}, \mathrm{b})=\sum \sqrt{ } \mathrm{a}(\mathrm{i}) \mathrm{b}(\mathrm{i}) €[0,1]$} histograms.Detected tumor part is segmented using active contour model[21].
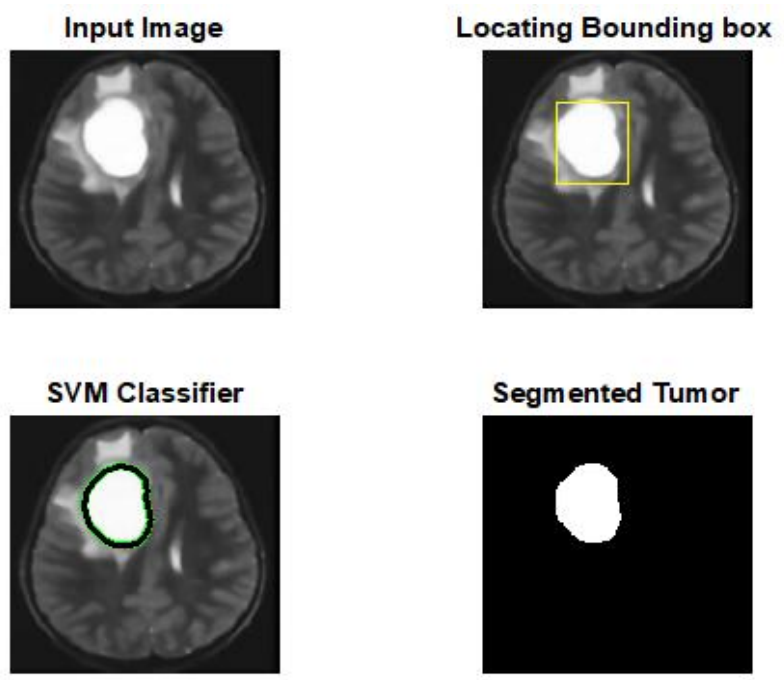

Fig.2 Segmented area from the input MRI image

\section{F. Edge Detection}

to distinguish the limit of the photo pixels inside the picture, it offers the element attributes of the photograph for department. that is also can undergo in thoughts as a vector incorporates length of the inclination and heading of an side. The extraction of highlights desires to diminish the quantity of records in a photo and the essential auxiliary attributes were applied to manner the the rest of the photo. on this proposed technique channel the district of hobby(ROI) likewise understand the contaminated encompassed walls in the mind photograph. This technique improves and improvements the managing time because the problem making ready calculation going to apply for distinguished areas, not into the complete image outline. on this aspect area step first, comply with the vector subtraction calculation next ROI is distinguished via manner of locating the comparative neighboring factors within the resultant bits in the resultant photo from the vector subtraction[21].

\section{G.Category}

The real ability of the affiliation step is to combination the comparative highlights of statistics thoughts MRI photo pixels depending on the recovered authentic highlights in the issue extraction venture of the photo. In AI there are forms of grouping, one is managed characterization another is solo affiliation. Regulated association procedures to prepare with the aid of the highlights of getting prepared data, in view of those organized highlights the check statistics has been predicted and study the version. Be that as it can, in unaided
Where $a(i)$ and $b(i)$ are the two normalized

gaining knowledge of method, the model has not been required making equipped set depending at the highlights the example has been expected. There are some commands of Supervised arrangement, they may be recorded right here

- artificial/coherent methods: selection timber

- Perceptron based totally strategies: single layered perceptron, Multilayered PerceptronRadial foundation potential structures.

- Statistical systems: Naive Bayes, Bayesian structures,

- okay-Nearest neighbor classifiers.

- assist Vector Machines.

on this paper, we've proposed the directed characterization machine is aid Vector device to installation the MRI picture pixels are regular or anomalous.

\section{H. Bolster Vector device}

SVM is an effective grouping calculation, it is applied for awesome undertakings, as an example, sincere and direct affiliation assignments, however it more and more complicated of nonlinear characterization issues are understood. In SVM hyperplane to isolate the precise elegance limits of statistics focuses, that is the key hobby of SVM grouping. In direct and nonlinear times treated all troubles of detachable and no fantastic with the aid of SVM. The proposed grouping hassle is to isolate the tumor and ordinary image from the given MRI image so SVM hyperplane to isolate the standard and uncommon cerebrum photographs relying on the given information and skills.SVM begins an order before everything with the aid of discriminative highlights at that component embody much less discriminative highlights
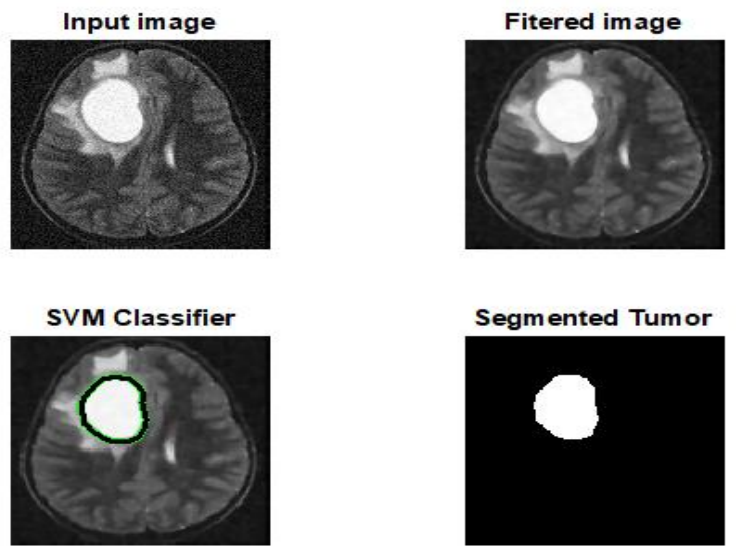

Fig.3 SVM classifier

GLCM and IBF strategies were removed the ideal highlights, include choice done by PCA strategy and it is given the contribution of SVM[22] classifier. Contrast and ordinary classifier SVM deals with the enormous element spaces and better speculation properties. Bigger capabilities were given as the contribution of SVM classifier so auxiliary misclassification hazard has been decreased at the season of preparing stage. Here we are utilized double arrangement in light of the fact that the picture was grouped dependent on two classes they are ordinary and unusual pixels. We are proposed SVM classifier piece capacity is spiral premise function(RBF)kernel. From the arrangement of $\mathrm{N}$ tests, the

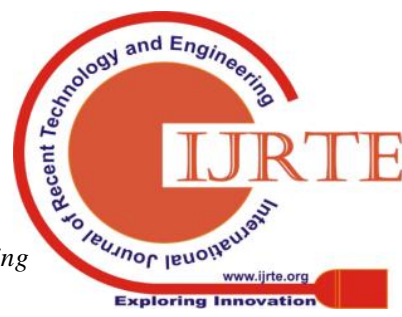


some of them as preparing and remaining are trying. To improve the exactness of the model, same strategies were rehashed until showing signs of improvement precision.

\section{III.RESULTS AND DISCUSSION}

The database consists of brain MRI images, an automatic model for detecting tumor to test the various patient image features has been extracted, analyzed and classified whether it has affected or not. The system has been implemented in MATLAB R2017a and SVM as the classifier.

The model result has been evaluated based on the following parameters sensitivity, specificity, and accuracy.

Patient: Tumor positive

Healthy: Tumor negative

True positive $(\mathbf{T P})=$ No of cases identified Tumor correctly

False positive $(\mathbf{F P})=$ No of cases wrongly identified of tumor patients

True negative $(\mathbf{T N})=$ No of cases identify the healthy images correctly

False negative $(\mathbf{F N})=$ No number of cases incorrectly identified as normal

The sensitivity of a model is its capability to find out the patient cases correctly. To calculate it, we should calculate the fraction of TP in patient cases. Mathematically, this can be represented as:

$$
\text { Sensitivity }=\frac{T P}{T P+F N}
$$

The specificity of a model is its capability to find the normal cases correctly. To calculate it, we should calculate the fraction of true negative in normal cases. Mathematically, this can be represented as:

$$
\text { Specificity }=\frac{T P}{T N+F P}
$$

The accuracy of a model is its ability to categorize the tumor and normal cases correctly. To calculate the accuracy of a test, we should calculate the proportion of true positive and true negative in all evaluated cases. Mathematically, this can be represented as:

$$
\text { Accuracy }=\frac{T P+T N}{T P+T N+F P+F N}
$$

After running the model our system achieved,

\begin{tabular}{|l|l|l|l|}
\hline S.No. & Accuracy & Sensitivity & Specificity \\
\hline 1 & $96.2 \%$, & $97 \%$, & $94.1 \%$. \\
\hline
\end{tabular}

\section{CONCLUSION}

We had built up a version for programmed discovery tumor from the human MRI pictures using AI technique. This model characterizes and segregates amongst everyday and unusual MRIs of the cerebrum utilizing SVM classifier. From the pre-prepared pictures, highlights had been separated using GLCM,IBF and highlights are chosen thru the approach for PCA.FBB department strategy and SVM classifier used to symbolize and element the tumor from the info MRI pictures.The approach were given $96.51 \%$ grouping precision on the cerebrum MRI image datasets. in addition studies is probably advanced by means of augmenting the exploratory sufferers to attain at an increasingly more actual, extra grounded measurable centrality end result.

\section{REFERENCES}

1. L. Guo, L. Zhao, Y. Wu, Y. Li, G. Xu, and Q. Yan, "Tumor identification in MR snap shots utilising one-magnificence resistant difficulty weighted SVMs," IEEE Transactions on Magnetics, vol. forty seven, no. 10, pp. 3849-3852, 2011. View at writer • View at Google pupil $\bullet$ View at Scopus.

2. R. Kumari, "SVM order a way on identifying irregularity in mind MRI images," global mag of Engineering research and applications, vol. three, pp. 1686-1690, 2013. View at Google student.

3. R. Rana, H. S. Bhadauria, and A. Singh, "research of different strategies for cerebrum tumor branch from MRI photos", global journal of growing generation and advanced Engineering, vol. three, no. 2, pp.338-342, 2013.

4. J. Joshi, et. al., "highlight Extraction and Texture category in MRI" In unique trouble of IJCCT, pp. 130-136, 2010.

5. Shan Shen, William Sandham, 2005 , " MRI Fuzzy Segmentation of thoughts Tissue using community attraction With Neural-community Optimization ", IEEE exchanges on statistics innovation in biomedicine volume 9, difficulty no.3,pp. 459 - 467.

6. Chaplot, S., L. M. Patnaik, and N. R. Jagannathan, "Order of appealing reverberation cerebrum images using wavelets as contribution to assist vector system and neural tool," Biomedical signal Processing and manipulate, Vol. 1, No. 1, 86-92, 2006.

7. Cocosco, C. An., A. P. Zijdenbos, and A. C. Evans, "a totally programmed and strong cerebrum MRI tissue characterization technique," medical photograph evaluation, Vol. 7, No. 4, 513-527, 2003.

8. Zhang, Y. moreover, L. Wu, "masses enhancement of neural system thru progressed BCO method," improvement In Electromagnetics studies, Vol. eighty three, 185-198, 2008.

9. Yeh, J.- Y. what is greater, J. C. Fu, "A severa leveled hereditary calculation for branch of multi-ghostly human-thoughts MRI," expert systems with programs, Vol. 34, No. 2, 1285-1295, 2008.

10. Patil, N. S., et al., "Relapse models using instance are looking for helped least square help vector machines," Chemical Engineering studies and layout, Vol. eighty three, No. eight, 1030-1037, 2005.

11. Wang, F.- F. what's greater, Y.- R. Zhang, "The assist vector device for dielectric target popularity thru a divider," progress In Electromagnetics research Letters, Vol. 23, 119-128, 2011.

12. $\mathrm{Xu}, \mathrm{Y} ., \mathrm{Y}$. Guo, L. Xia, and $\mathrm{Y}$. Wu, "A assist vector relapse primarily based completely nonlinear demonstrating technique for Sic mesfet," development In Electromagnetics studies Letters, Vol. 2, 103-114, 2008.

13. Li, D., W. Yang, and S. Wang, "Order of far flung strands in cotton constructing up the use of system imaginative and prescient and multi-elegance bolster vector system," computers and Electronics in Agriculture, Vol. 4, No. 2, 274-279, 2010

14. R. Ahmmed and M. F. Hossain, "Tumor degrees identification in thoughts MRI photograph utilizing Temperbased good enough-implies and Fuzzy C-implies Clustering set of policies", intending of eleventh global Engineering, technology and generation convention, pp. 1-10, 18 - 19 December, 2015, BIAM foundation, Dhaka.

15. O.P. Verma, M. Hammandlu, S. Susan, M. Kulkami and

P.ok. Jain, "A fundamental unmarried 
seeded district developing calculation for shading image branch utilising versatile thresholding," 2011 worldwide conference on conversation structures and network technology,@2011 IEEE.

16. M. Lugina, N. D. Retno, and R. Rita, "mind Tumor Detection and kind in Magnetic Resonance Imaging (MRI) using place growing, Fuzzy Symmetric diploma, and artificial NeuralNetwork returned proliferation", worldwide magazine on ICT, vol. 1, pp. 20-28,December, 2015

17. Baidya Nath Saha ,Nilanjan Ray,Russel Greiner ,Albert Murtha,Hong Zhang "speedy discovery of thoughts tumors and edemas: A bouncing container strategy using symmetry", comput Med Imag.and Graph, Vol. 36, No.2, pp. ninety five $-107,2012$.

18. T. F Chan and L. Vese, "A functioning form version with out edges", Cpp. 141 - 151,1999.

19. Haralick R.M., Shanmugam okay. what is more, Dinstein I., "Textural capabilities for image classification," IEEE Trans. on systems, guy and Cybernetics three(6),1973, pp 610-621.

20. Saha, B.; Ray, N.; Greiner, R.; Murtha, A.; Zhang, H. fast discovery of cerebrum tumors and edemas: A bouncing subject method using symmetry. Comput. drugs. Imaging Graph. 2012, 36, ninety five-107.

21. Zhang KH, Zhang L, track HH, Zhou WG (2010) lively shapes with specific community or international division: another detailing and stage set approach. image and vision Computing 28: 668-676.

22. S. C. Chen and D. Q. Zhang, - sturdy image department utilizing FCM with spatial necessities depending on new Kernel - induced separation degree,\| IEEE Transactions on systems, guy, and Cybernetics, element B (Cybernetics), vol. 34, issue 4, pp. 1907-1916, 2004.

23. Y. Zhang and $\mathrm{L} \mathrm{Wu}, \mathrm{A} \mathrm{Mr}$ thoughts pics Classifiervia fundamental aspect assessment And KernelSupport Vector tool, improvement InElectromagnetics research, VoL a hundred thirty, 369,388, 2012.

24. Haralick, R.M., Shanmugam, k., Dinstein, Its'Hak: Textural capabilities for photograph Classication. IEEE Transactions on structures, guy and Cybernetics SMC-three (1973) 610-621

25. Jaffar, Arfan and Zia, Sultan and Latif, Ghazanfar and Mirza, Anwar and Mehmood, Irfan and Ejaz, Naveed and Baik, Sung. (2012). Anisotropic Diffusion primarily based definitely mind MRI Segmentation and 3-d Reconstruction. international magazine of Computational Intelligence five.10.1080/18756891.2012.696913.

systems.

26. Roy, S., and Maji, P. (2015). A simple skull stripping calculation for thoughts MRI. 2015 8th worldwide conference on Advances in sample recognition (ICAPR). 